\title{
Assessing the Impact of Gestational Age of Donors on the Efficacy of Amniotic Epithelial Cell-Derived Extracellular Vesicles in Experimental Bronchopulmonary Dysplasia
}

Tamara Yawno ( $\sim$ tamara.yawno@monash.edu )

Monash University Department of Paediatrics https://orcid.org/0000-0002-4257-3814

Dandan Zhu

Hudson Institute of Medical Research

Mirja Krause

Hudson Institute of Medical Research

Gina Kusuma

Hudson Institute of Medical Research

Renate Schwab

Hudson Institute of Medical Research

Mehri Barabadi

Hudson Institute of Medical Research

Amina S Maleken

Hudson Institute of Medical Research

\section{Siow Chan}

Hudson Institute of Medical Research

Rod Hunt

Monash University Department of Paediatrics

David Greening

Baker IDI: Baker Heart and Diabetes Institute

Euan Wallace

Monash University

Rebecca Lim

Hudson Institute of Medical Research

\section{Research Article}

Keywords: human amniotic epithelial cells, extracellular vesicles, prematurity, bronchopulmonary dysplasia 
Posted Date: February 15th, 2022

DOI: https://doi.org/10.21203/rs.3.rs-1340653/v1

License: (c) (i) This work is licensed under a Creative Commons Attribution 4.0 International License. Read Full License 


\section{Abstract}

Background and rationale: Extracellular vesicles (EVs) are a potential cell-free regenerative medicine. Human amniotic epithelial cells (hAECs) are a viable source of cell therapy for diseases like bronchopulmonary dysplasia (BPD). However, little is known about the impact of gestational age of the donor on the quality of hAEC-derived EVs.

Aims: To determine the impact of gestational age on hAEC-derived EVs in experimental BPD.

Results: Term hAEC-derived EVs displayed a significantly higher density of surface epitopes (CD142 and CD133) and induced greater macrophage phagocytosis compared to preterm hAEC-EVs. However, T cell proliferation was more significantly suppressed by preterm hAEC-EVs. Using a model of experimental BPD, we observed that term but not preterm hAEC-EVs improved tissue-to-airspace ratio and septal crest density. While both term and preterm hAEC-EVs reduced the levels of inflammatory cytokines on postnatal day 7 , the improvement of lung injury was associated with increased type II alveolar cells which was only observed in term hAEC-EV treatment group. Furthermore, only neonatal term hAEC-EVs reduced airway hyper-responsiveness, mitigated pulmonary hypertension and protected against right ventricular hypertrophy at 6 weeks of age.

Conclusion: Term hAEC-EVs, but not preterm hAEC-EVs, have therapeutic efficacy in a mouse model of BPD-like lung injury. Therefore, the impact of donor criteria should be considered when applying perinatal cells derived EV therapy for clinical use.

\section{Background}

Extracellular vesicles (EVs) derived from pro-regenerative cells are being considered as an alternative for regenerative medicine, serving as non-living carriers of therapeutic proteins, lipids, mRNA and microRNA ${ }^{1}$. At the time of this manuscript preparation, 31 clinical trials have been registered with the National Institute of Health $(\mathrm{NIH})$, investigating EV-based therapeutics (https://clinicaltrials.gov). These include 5 trials (NCT02138331, NCT03437759, NCT04213248, NCT04798716, NCT04134676) using EVs isolated from umbilical cord-blood or umbilical cord derived mesenchymal stromal/stem cells (UC-MSCs).

Perinatal stem/stem-like cells derived from gestational tissues include a few regenerative cell types such as hematopoietic stem/progenitor cells (HSCs/HPCs), endothelial progenitor cells (EPCs), mesenchymal stem/stromal cells (MSCs) and human amniotic epithelial cells (hAECs) ${ }^{2}$. Recent studies have shown that MSCs and hAECs are promising cell therapies for bronchopulmonary dysplasia (BPD) ${ }^{3}$. There are now $12 \mathrm{NIH}$ registered clinical trials using UC-MSCs as a treatment for BPD (https://clinicaltrials.gov).

The therapeutic effects of perinatal stem cells from preterm birth remain a subject of debate. UC-MSCs from preterm birth were shown to have beneficial effects in treating lung injury ${ }^{4}$, however, others found that preterm cord blood cells were less effective in treating preterm brain injury compared to term cord blood cells ${ }^{5}$. There has been limited information to date on how gestational age influences EV quality and 
potency. EVs derived from UC-MSCs have been shown to have therapeutic effects in experimental models of BPD. Kourembanas et al discovered that MSC-EVs derived from term umbilical cord Wharton's jelly improved lung function and ameliorated secondary pulmonary hypertension ${ }^{6}$. Bhandari et al found that MSC-EVs derived from early gestational age umbilical cord also improved lung structure and reduced inflammation in a mouse model of $\mathrm{BPD}^{4}$. Lim et al suggested that there was an inverse correlation between the MSC-EV production and developmental maturity of the donor tissues, however, this did not affect the efficacy ${ }^{7}$. It is therefore important to understand if and how the gestational age of the donor influences the yield and potency of EVs derived from perinatal stem/stem-like cells.

The anti-inflammatory and anti-fibrotic effects of term hAECs in lung repair have been previously shown in several mouse and sheep lung injury models ${ }^{8-10}$. hAECs play several important roles in maternal-fetal tolerance during pregnancy, inducing expansion of regulatory $T$ cells and limit chronic inflammatory diseases ${ }^{11}$, polarise macrophages from classical M1 to alternative M2 phenotypes, and encourage phagocytic activity ${ }^{12}$. Recently, hAECs have been shown to have potent immunomodulatory properties associated with their constitutive production of ectoenzymes that are able to drive expansion of regulatory $B$ cells and suppress NK cell activity ${ }^{13}$. hAECs from term, healthy pregnancies appear to be a potent source of cell therapy for $\mathrm{BPD}^{14}$, which is a major respiratory complication associated with extreme prematurity. Most recently, hAECs have progressed into clinical trials for BPD ${ }^{15-17}$.

Little is known about the impact of gestational age of the donor on EVs derived from hAECs (hAEC-EVs). To address this gap in research, we used a combination of in vitro potency assays and murine model of experimental BPD ${ }^{18}$ to: 1) characterise term and preterm hAEC-EVs; and 2) compare the therapeutic potential of term hAECs, term hAEC-EVs and preterm hAEC-EVs in experimental BPD.

\section{Methods}

\section{hAEC Isolation}

hAECs were isolated from amnions collected after caesarean sections and cell purity was determined by flow cytometry as previously described ${ }^{19}$. Cell viability was determined using Trypan blue exclusion, where cells of $>80 \%$ viability were used in this study. The mean gestational age of term donors was 38 weeks \pm 4 days and 33 weeks \pm 2 days for preterm donors. All isolated cell lines were de-identified in accordance with guidelines on human research ethics as set out by the National Health and Medical Research Council.

\section{hAEC-EV Isolation}

Ten million hAECs were plated into a T175 flask with $25 \mathrm{~mL}$ UltraCULTURE ${ }^{\mathrm{TM}}+1 \% \mathrm{~L}$-glutamine serum-free media (UltraCULTURE ${ }^{\text {TM}}$; LONZA, U.S.A). Cells were cultured for four days at $37^{\circ} \mathrm{C}, 95 \%$ humidified air and $5 \% \mathrm{CO}_{2}$, and then the conditioned media was collected for $\mathrm{EV}$ isolation using serial ultracentrifugation as previously described ${ }^{20}$. 


\section{hAEC-EV Characterisation}

The characterisation of hAEC-EVs $(n=5)$ was performed in accordance with the Minimal Information for Studies of Extracellular Vesicles (MISEV) guidelines ${ }^{21}$. The morphology of hAEC-EVs were determined by transmission electron microscopy (TEM). Protein concentration was determined using a bicinchoninic acid (BCA) assay (Pierce BCA Protein Assay Kit, Thermo Fisher Scientific, U.S.A). Nanoparticle tracking analysis (NTA, NanoSight, U.K) was used to measure particle numbers and size distribution. Surface epitopes were determined using the MACSPlex Exosome kit (130-108-813, Miltenyi Biotec, U.S.A). Samples were processed according to each manufacturer's instructions. Proteome composition of hAECEVs was determined using proteomic analysis as previously described ${ }^{22}$.

\section{T Cell Proliferation Assay}

Freshly isolated CD4 ${ }^{+}$human T cells were labelled with CellTrace Violet (CTV, Thermo Fisher Scientific, U.S.A), activated with Dynabeads (Thermo Fisher Scientific, U.S.A) coated with human anti-CD3/CD28 (beads:cell =1:1), and cultured with/without hAEC-EVs for 96 hours before analysed with fluorescenceactivated single cell sorting (FACS analysis).

\section{Macrophage Phagocytosis Assay}

Immortalized monocyte-like cells, THP-1, were stimulated with paramethoxyamphetamine (PMA, $200 \mathrm{nM} / \mathrm{mL}$ ) for 72 hours before hAEC-EVs were added and incubated for another 24 hours. pHrodo Red E. coli BioParticles (100ug/mL, Thermo Fisher Scientific, U.S.A) was added and incubated for 2 hours, and then the fluorescent intensity was measured at $560 / 585 \mathrm{~nm}$.

\section{Animals and Experimental Groups}

C57/BL6 mice were time-mated and used for these experiments. Mice were kept in 12h light/dark cycle and had free access to water and food. On day 16 of pregnancy (embryonic; E16), mice were injected with either $0.1 \mu \mathrm{g}$ lipopolysaccharide (LPS; Sigma, St. Louis, MO) in $5 \mu \mathrm{L}$ saline or an equal volume of vehicle into each amniotic sac. Pups were then allowed to deliver naturally at term (E21). Newborn pups were exposed to either normoxia (Fraction of Inspired Oxygen; FiO2 $=0.2$ ) or hyperoxia $(F i O 2=0.65$ ) from postnatal day 3.5 (PND3.5). On PND4, pups received either term hAECs (the previously shown effective therapy ${ }^{23}$ ), term hAEC-EVs, preterm hAEC-EVs, or saline via intravenous delivery through the superficial temporal vein. For short term studies, mouse pups were culled on PND 7 and 14. For long term studies, pups were kept in hyperoxia chamber until PND28 when they were weaned and transferred to standard housing facilities, before experiments were terminated at 6 weeks of age. To prevent maternal oxygen toxicity, nursing dams were rotated between chambers every two days. A representation of the experimental design is shown in Fig. 1. Term and preterm hAEC-EVs used in animal work were pooled from 5 cell lines each. 


\section{Experimental groups:}

\section{a. Intra-amniotic saline + normoxia + intravenous saline,}

\section{b. Intra-amniotic LPS + hyperoxia + intravenous saline,}

\section{c. Intra-amniotic LPS + hyperoxia + intravenous term hAECs $(100,000$ cells),}

\section{d. Intra-amniotic LPS + hyperoxia + intravenous term hAEC- EVs (10 mg),}

\section{e. Intra-amniotic LPS + hyperoxia + intravenous preterm hAEC-EVs (10mg).}

\section{Lung Histology and Immunohistochemistry Staining}

Mouse pup lungs were inflated and fixed with $4 \%(\mathrm{w} / \mathrm{v})$ paraformaldehyde. Paraffin sections were cut at $5 \mu \mathrm{m}$ thickness and stained with haematoxylin and eosin (H\&E). Eight to ten non-overlapping sequential fields of view were taken at 200x magnification. Lung development and alveolar simplification were determined by measuring tissue-to-airspace ratio as previously described ${ }^{24}$. Hart's staining and immunohistochemistry, von Willebrand Factor (vWF), alpha-smooth muscle actin (a-SMA), bronchioalveolar stem cell (BASC) and type II alveolar cells (AT2s) were performed and image analysis completed as previously described ${ }^{23-25}$. The ratio of secondary septal crests to tissue was calculated, pulmonary vessel numbers were determined by counting the number of vWF-positive vessels stratified by diameter ( $\leq 50$ and $>50 \mu \mathrm{m}$ ), and the average fluorescence intensity of a-SMA by perimeter for each pulmonary artery was calculated using Image $\mathrm{J}(\mathrm{NIH}$, Bethesda, MD). The average number of BASCs per terminal bronchiole was determined by manual counting while the percentage number of AT2s was determined using Imaris software (Bitplane, Zurich, Switzerland).

\section{Lung Lysate Levels of Inflammatory Cytokines}

Assessment of inflammatory cytokine concentration was performed on lung lysates using a multiplex an enzyme-linked immunosorbent assay (ELISA) for murine interleukin (IL)-1 $\beta, \mathrm{IL}-6$, tumour necrosis factoralpha (TNF-a), monocyte chemoattractant protein (MCP)-1, macrophage inflammatory protein (MIP)-2, leukemia inhibitory factor (LIF), granulocyte-macrophage colony-stimulating factor (GM-CSF), and regulated upon activation, normal T cell expressed and presumably secreted (RANTES) (Bio-rad, CA, USA). The levels of cytokines were normalised against total protein concentration. 


\section{Physiological Measurements}

At postnatal weeks 6, invasive plethysmography was performed using the flexiVent system (SCIREQ, Montreal, QC, Canada) as described before ${ }^{23}$. Changes to respiratory system resistance (Rrs), compliance (Crs), and pressure-volume loop (PV loop) were measured. Transthoracic echocardiography was also performed using a Vevo 2100 (Visualsonics, Toronto, Canada) as described previously ${ }^{23}$. Changes to the pulmonary artery acceleration time (PAT), the pulmonary artery ejection time (PET) and right ventricle anterior wall thickness (RVAWT) were measured.

\section{Statistical Analysis}

Data were expressed as mean \pm standard error of mean (SEM). Statistical significance was determined with one-way analysis of variance (AVONA) accompanied by the Bonferroni post-hoc test for multiple groups or the Mann-Whitney test when comparing between two groups. Statistical significance was accorded when $\mathrm{p}<0.05$.

\section{Results}

\section{Isolation and Characterisation of Term and Preterm hAEC- EVs}

hAEC-EVs typically exhibited a cup-shaped morphology across all cell lines regardless of gestational age of the donor (Fig. 2A). We did not observe significant differences in the cell viability, EV protein yield, EV particle number, particle mean size and size distribution between term and preterm hAECs (Fig. 2B-F).

hAEC-EVs displayed tetraspanins common to EVs, namely CD9, CD81 and CD63, as well as soluble vascular endothelial growth factor (VEGF) receptor CD105, and epithelial cell marker CD326. There were no differences in expression levels of these surface epitopes between term and preterm hAEC-EVs (Fig. 3A-D). However, term hAEC-EVs displayed higher levels of CD142 and CD133 compared to preterm hAECEVs (Fig. 3E-F).

Proteomic analysis confirmed that term and preterm hAEC-EV samples contained EV tetraspanins such as CD9 and CD81, as well as other EV markers such as Alix, heat shock proteins (HSP)70, and syntenin binding protein (SDCBP). EV-exclusion markers such apolipoproteins (APO) A1, APOAB, and albumin (ALB) were not detected in any hAEC-EV samples (data not shown). The 47 proteins were more than 20fold higher in term hAEC-EVs (Table 1), while 35 proteins were more than 20 -fold higher in preterm hAECEVs (Table 2). Pathway analysis of proteins in Tables 1 and 2 were shown in Fig. 4A and 4B respectively. 
Table 1

47 proteins with $>20$-fold change in term hAEC-EVs compared to preterm hAEC-EVs

\begin{tabular}{|c|c|c|}
\hline Protein names & Gene names & $\operatorname{LogFC}$ \\
\hline Mucin-16 & MUC16 CA125 & 26.3 \\
\hline Alpha-2-macroglobulin-like protein 1 & A2ML1 CPAMD9 & 25.3 \\
\hline Collagen alpha- $1(X V I I)$ chain & COL17A1 BP180 BPAG2 & 25.3 \\
\hline SPARC & SPARC ON & 25.2 \\
\hline Prostaglandin F2 receptor negative regulator & $\begin{array}{l}\text { PTGFRN CD9P1 EWIF FPRP } \\
\text { KIAA1436 }\end{array}$ & 25.0 \\
\hline Collagen alpha-1(XII) chain & COL12A1 COL12A1L & 24.9 \\
\hline Syntenin-2 & SDCBP2 SITAC18 & 24.8 \\
\hline Syntaxin-4 & STX4 STX4A & 24.8 \\
\hline Small proline-rich protein 3 & SPRR3 SPRC & 24.6 \\
\hline Sorcin & SRI & 24.4 \\
\hline Monocarboxylate transporter 4 & SLC16A3 MCT4 & 24.4 \\
\hline Synaptosomal-associated protein 23 & SNAP23 & 24.1 \\
\hline F-actin-capping protein subunit alpha-1 & CAPZA1 & 24.0 \\
\hline Serine protease 23 & PRSS23 ZSIG13 UNQ270/PR0307 & 23.8 \\
\hline Unconventional myosin-lc & MY01C & 23.7 \\
\hline Catenin beta-1 & $\begin{array}{l}\text { CTNNB1 CTNNB OK/SW-cl.35 } \\
\text { PRO2286 }\end{array}$ & 23.7 \\
\hline Cystatin-M & CST6 & 23.6 \\
\hline Protein S100-A13 & S100A13 & 23.5 \\
\hline Antithrombin-III & SERPINC1 AT3 PR00309 & 23.5 \\
\hline Hemoglobin subunit beta & HBB & 23.3 \\
\hline Syndecan-4 & SDC4 & 23.2 \\
\hline Cytoplasmic FMR1-interacting protein 1 & CYFIP1 KIAA0068 & 23.2 \\
\hline Radixin & RDX & 23.1 \\
\hline Plasminogen activator inhibitor 1 & SERPINE1 PAI1 PLANH1 & 23.0 \\
\hline Monocarboxylate transporter 1 & SLC16A1 MCT1 & 23.0 \\
\hline
\end{tabular}




\begin{tabular}{|c|c|c|}
\hline Protein names & Gene names & LogFC \\
\hline $\begin{array}{l}\mathrm{N}(\mathrm{G}), \mathrm{N}(\mathrm{G}) \text {-dimethylarginine dimethylaminohydrolase } \\
2\end{array}$ & DDAH2 DDAH G6A NG30 & 22.8 \\
\hline Choline transporter-like protein 1 & SLC44A1 CD92 CDW92 CTL1 & 22.8 \\
\hline Tissue factor & F3 & 22.7 \\
\hline Catenin delta-1 & CTNND1 KIAA0384 & 22.7 \\
\hline Gap junction beta-3 protein & GJB3 CX31 & 22.5 \\
\hline Tropomyosin alpha-1 chain & TPM1 C15orf13 TMSA & 22.5 \\
\hline Glutathione S-transferase omega-1 & GST01 GSTTLP28 & 22.4 \\
\hline Putative annexin A2-like protein & ANXA2P2 ANX2L2 ANX2P2 LPC2B & 22.4 \\
\hline F-actin-capping protein subunit beta & CAPZB & 22.3 \\
\hline Lysosome-associated membrane glycoprotein 1 & LAMP1 & 22.3 \\
\hline $\begin{array}{l}\text { Brain-specific angiogenesis inhibitor 1-associated } \\
\text { protein } 2\end{array}$ & BAIAP2 & 22.1 \\
\hline Syntaxin-binding protein 3 & STXBP3 & 21.8 \\
\hline Serine/threonine-protein phosphatase CPPED1 & CPPED1 CSTP1 & 21.8 \\
\hline Plakophilin-2 & PKP2 & 21.7 \\
\hline Src substrate cortactin & CTTN EMS1 & 21.7 \\
\hline Charged multivesicular body protein $4 \mathrm{~b}$ & CHMP4B C20orf178 SHAX1 & 21.6 \\
\hline Protein piccolo & PCLO ACZ KIAA0559 & 21.5 \\
\hline Ras-related protein R-Ras & RRAS & 21.4 \\
\hline Laminin subunit gamma-1 & LAMC1 LAMB2 & 21.3 \\
\hline $\begin{array}{l}\text { Guanine nucleotide-binding protein } G(i) \text { subunit } \\
\text { alpha-1 }\end{array}$ & GNAl1 & 21.3 \\
\hline Annexin A13 & ANXA13 ANX13 & 20.6 \\
\hline Glutaredoxin-3 & GLRX3 PICOT TXNL2 HUSSY-22 & 20.4 \\
\hline
\end{tabular}


Table 2

35 proteins with $>20$-fold change in preterm hAEC-EVs compared to term hAEC-EVs

\begin{tabular}{|c|c|c|}
\hline Protein names & Gene names & LogFC \\
\hline Protein kinase $\mathrm{C}$ & PACSIN1 KIAA1379 & 26.9 \\
\hline Myosin light chain 3 & MYL3 & 25.1 \\
\hline Myosin-6 & MYH6 MYHCA & 24.7 \\
\hline Histone H2AX & H2AFX H2AX & 23.9 \\
\hline Barrier-to-autointegration factor & BANF1 BAF BCRG1 & 23.1 \\
\hline Malate dehydrogenase & MDH1 MDHA & 22.9 \\
\hline Fructose-bisphosphate aldolase $\mathrm{C}$ & ALDOC ALDC & 22.9 \\
\hline Complement factor $\mathrm{H}$ & CFH HF HF1 HF2 & 22.6 \\
\hline Heterogeneous nuclear ribonucleoproteins A2/B1 & HNRNPA2B1 HNRPA2B1 & 22.4 \\
\hline Transaldolase & TALD01 TAL TALDO TALDOR & 22.4 \\
\hline Dihydropyrimidinase-related protein 2 & DPYSL2 CRMP2 ULIP2 & 22.4 \\
\hline Glycogen phosphorylase, liver form & PYGL & 22.3 \\
\hline ATP synthase subunit alpha & $\begin{array}{l}\text { ATP5F1A ATP5A ATP5A1 } \\
\text { ATP5AL2 ATPM }\end{array}$ & 22.1 \\
\hline Rab GDP dissociation inhibitor alpha & $\begin{array}{l}\text { GDI1 GDIL OPHN2 RABGDIA } \\
\text { XAP4 }\end{array}$ & 22.1 \\
\hline Proteasome subunit alpha type-3 & PSMA3 HC8 PSC8 & 22.0 \\
\hline E3 ubiquitin-protein ligase TRIP12 & TRIP12 KIAA0045 ULF & 22.0 \\
\hline Serine/arginine-rich splicing factor 2 & SRSF2 SFRS2 & 21.9 \\
\hline 1-phosphatidylinositol & PLCD1 & 21.8 \\
\hline Histone H1.0 & H1F0 H1FV & 21.8 \\
\hline $60 S$ acidic ribosomal protein $\mathrm{P} 2$ & RPLP2 D11S2243E RPP2 & 21.5 \\
\hline $60 S$ acidic ribosomal protein $\mathrm{PO}$ & RPLPO & 21.5 \\
\hline Malate dehydrogenase & $\mathrm{MDH} 2$ & 21.4 \\
\hline $\begin{array}{l}\text { Guanine nucleotide-binding protein } \mathrm{G}(\mathrm{s}) \text { subunit alpha } \\
\text { isoforms short }\end{array}$ & GNAS GNAS1 GSP & 21.4 \\
\hline Transitional endoplasmic reticulum ATPase & VCP & 21.3 \\
\hline Proteasome subunit alpha type-7 & PSMA7 HSPC & 21.2 \\
\hline
\end{tabular}




\begin{tabular}{|lll|}
\hline Protein names & Gene names & LogFC \\
\hline Proteasome subunit alpha type-6 & PSMA6 PROS27 & 21.1 \\
\hline Nuclease-sensitive element-binding protein 1 & YBX1 NSEP1 YB1 & 20.9 \\
\hline Epithelial membrane protein 3 & EMP3 YMP & 20.8 \\
\hline Actin-related protein 2/3 complex subunit 4 & ARPC4 ARC20 & 20.6 \\
\hline Dipeptidyl peptidase 4 & DPP4 ADCP2 CD26 & 20.5 \\
\hline Syntenin-1 & SDCBP MDA9 SYCL & 20.5 \\
\hline Phosphoglucomutase-1 & PGM1 & 20.3 \\
\hline Cytoplasmic dynein 1 intermediate chain 2 & DYNC1I2 DNCI2 DNCIC2 & 20.2 \\
\hline Heterogeneous nuclear ribonucleoprotein D0 & HNRNPD AUF1 HNRPD & 20.0 \\
\hline Ubiquitin-like protein 3 & PACSIN1 KIAA1380 & 20.0 \\
\hline
\end{tabular}

\section{Comparative in vitro potency of term versus preterm hAEC- EVs}

The ability of term and preterm hAEC-EVs to directly influence the behaviour of T cells and macrophages was assessed. Here, we observed that while both term and preterm hAEC-EVs suppressed T cell proliferation, greater suppression was achieved with preterm hAEC-EVs (Fig. 5A). In contrast, term hAECEVs, but not preterm hAEC-EVs, significantly increased macrophage phagocytosis (Fig. 5B). These findings suggest that differences in EV cargo may be related to differential effects on target cells.

\section{Term hAEC-EVs Ameliorated Alveolar Simplification}

The combination of intra-amniotic LPS and neonatal hyperoxia significantly reduced tissue-to-air space ratio by PND 7 and 14 ( $p<0.001$ and $p<0.01)$ compared to control groups (Fig. 6A-C). Only term hAECEVs improved tissue-to-air space ratio such that they were not significantly different to the injured group and hAEC treatment group by PND7 (Fig. 6B). Furthermore, term hAEC-EVs mitigated the injury by PND14 as seen by the improvement in tissue-to-air space ratio, such that they were comparable to the control group (Fig. 6A, 6C). In contrast, tissue-to-air space ratio of animals given preterm hAEC-EVs remained significantly lower than healthy controls $(p<0.001$, Fig. $6 \mathrm{~A}, 6 \mathrm{C})$. The tissue to airspace ratio achieved by the hAEC was higher than both hAEC-EV treatment groups by PND7 $(p<0.05)$. The outcomes achieved by either hAECs or term hAEC-EVs were comparable by PND14 (Fig. 6B-C).

\section{Term hAEC-EV Treatment Improved Secondary Septal Crest Density}


Our model of experimentally induced BPD caused a decrease in septal crest density at PND7 and 14 compared to control animals (Fig. 7A-C). hAEC treatment in BPD animals significantly increased secondary septal crest density at PND7 and 14, such that they were comparable to control (Fig. 7B and C). Term but not preterm hAEC-EVs also increased septal crest density but only statistically significant by PND14 compared to BPD animals (Fig. 7C).

\section{Term hAEC-EV Treatment Decreased Lung Inflammation}

By PND7, both term and preterm hAEC-EV administration reduced IL-1 $\beta$ and TNF- $a$ to control levels $(p<$ 0.05 , Fig. $8 A-B$ ), which was comparable to hAEC treatment. Interestingly, higher levels of IL-1 $\beta$ and MIP-2 were observed in the group treated with preterm hAEC-EVs (Fig. 8C-D). The level of RANTES was elevated in hAEC treatment group, but not in the term hAEC-EV group $(p<0.05, p<0.01$, Fig. 8E). The levels of LIF, MCP-1 and GM-CSF were higher in the injury group. Both hAECs and term hAEC-EVs reduced them to control levels, but the levels of LIF and MCP-1 remained high in preterm hAEC-EV treated mice $\left({ }^{*} \mathrm{p}<0.05\right.$, ${ }^{* \star} p<0.01, * \star * p<0.001$, Fig. 8F-H). Levels of TNF- $a$ and IL- 6 were below the limit of detection.

\section{Term hAEC-EV Treatment Induced Type II Alveolar Cell but not Bronchioalveolar Stem Cell Proliferation}

Bronchioalveolar stem cells (BASCs) express both pro-SPC and CC10. They are located the terminal bronchioles. AT2s stained positive for pro-SPC and are located throughout the parenchyma (Fig. 9A). Experimentally induced BPD had no effect on BASC and AT2 cell activation. hAEC treatment significantly increased the average number of BASCs per terminal bronchiole compared to BPD and control animals by PND14. However, the average number of BASCs per terminal bronchiole did not change in response to either hAEC-EV group on both PND7 and 14 (Fig. 9B-C). However, the percentage of AT2 cells was significantly increased in the term hAEC-EV treatment group at both time points compared to control group $(p<0.05$ and $p<0.01)$. In contrast, there was no significant change in either preterm hAEC-EV or hAEC treatment groups (Fig. 9D-E).

\section{Term hAEC-EV Treatment Increased the number of vWF positive pulmonary vessels}

The number of small pulmonary vessels $(<50 \mu \mathrm{m}$ in diameter) were significantly decreased in the BPD group compared to controls on both PND7 and 14 ( $p<0.01$ and $p<0.05$, Fig. 10A-C). hAEC and term hAEC-EV treatment restored pulmonary vascularisation to control levels, but this was not achieved by preterm hAEC-EV treatment. (Fig. 10B-C). Larger blood vessels $(>50 \mu \mathrm{m})$ were unaffected across all experimental groups.

\section{Term hAEC-EV Treatment Reduced Peripheral Pulmonary Arterial Remodeling}

Pulmonary arterial hypertension caused by vascular remodelling is a common secondary complication to BPD. In order to assess this in experimentally induced BPD, the medial layer of the pulmonary arteries 
was stained for a-SMA (Fig. 11A). The thickness of the arterial medial layer was significantly increased in the experimental BPD group by PND14, but this was attenuated in the hAEC and term hAEC-EV treatment groups $(p<0.01, p<0.001, p<0.0001$, Fig. 11B).

\section{Term hAEC-EV Treatment Improved Lung Tissue-To- Airspace Ratio}

We observed that the reduced tissue-to-airspace ratio persisted until week 6 but was significantly improved by hAEC treatment. Neither term nor preterm hAEC-EVs significantly improved tissue-to-airspace ratio by PND14 (Fig. 12A-B)

\section{Term hAEC-EV Treatment Prevented the Increase of Airway Responsiveness}

Lung function test at postnatal week 6 showed that baseline respiratory system resistance (Rrs) and compliance (Crs) did not significantly change between groups (Fig. 13A-B). When challenged with $100 \mathrm{mg} / \mathrm{mL}$ methacholine, the injured mice showed significantly increased Rrs and decreased Crs compared to the control ( $p<0.001$ and $p<0.0001$ respectively, Fig. 13C-D). hAEC treatment restored Rrs and $\mathrm{Crs}$ to control levels. Term hAEC-EV treatment increased Crs to healthy mice level, and decreased Rrs to the level that was between control and injured groups $(p<0.05$, Fig. 13C-D). However, preterm hAECEVs did not mitigate airway response. The pressure-volume (PV) loop is generated by changes to lung volume during a respiratory cycle. Compared to control, the PV loop of the injured group saw a significant upward shift, indicating increased lung compliance and suggestive of reduced tissue elasticity. Both hAEC and term hAEC-EV treatment returned the loop downwards such that the PV loop sat between the control and injured groups. The PV loop position of mice that received preterm hAEC-EVs remained higher than control. (Fig. 13E).

\section{Term hAEC-EV Treatment Prevented Pulmonary Hypertension and Right Ventricular Hypertrophy}

Echocardiography showed that at postnatal week 6, the injury group developed pulmonary hypertension with reduced pulmonary artery acceleration to ejection time (PAT/PET, Fig. 14A-B), and right ventricle hypertrophy with thickened RVAW (Fig. 15A-B). These were attenuated by hAEC and term hAEC-EV treatment but not preterm hAEC-EV treatment (Fig. 14A-B, 15A-B).

\section{Discussion}

In this manuscript, we characterised term and preterm hAEC-EVs and assessed their efficacy in an experimentally induced BPD mouse model. This study provides the first evidence that hAEC-EVs from term and preterm pregnancy bore exosome characteristics based on size distribution, morphology and surface markers. We did, however, observe that EV cargo and functional potency between term hAEC-EVs 
and preterm hAEC-EVs were significantly different. Only term but not preterm hAEC-EVs were beneficial in the setting of BPD-like lung disease, which was comparable to the efficacy of term hAECs.

Both term and preterm hAEC-EVs appeared to be of the size range most commonly associated with exosomes, displaying their typical cup-shaped morphology with no significant difference in EV yield. In order to verify their identity, we sought to determine the presence of typical EV markers CD9, CD63, CD81, and ALIX. In current study, we observed EV yield from hAECs with 2 to $9 \mu \mathrm{g} \mathrm{EVs} \mathrm{from} 1 \times 10^{6}$ cells, or $4 \times 10^{10}$ to $24 \times 10^{10}$ particles between different hAEC donors, which may be due to the human biological variability. However, there was no significant difference in protein yield, particle concentration and particle size distribution between term and preterm hAEC-EVs. It is worth noting that others have reported yields of $10 \mu \mathrm{g} \mathrm{EVs}$ from $2.5 \times 10^{5}$ to $1 \times 10^{7}$ human bone marrow derived $\mathrm{MSCs}^{26,27 .}$

Further, when we examined exosomal surface epitopes, we have shown that term hAEC-EVs had higher level of CD142 and CD133. CD142 is also called tissue factor (TF), which belongs to the cytokine receptor class II family, and it is known to exist with membrane vesicles ${ }^{28}$. CD142 was proven to induce proangiogenic matrix for blood vessel infiltration, and the activation of TF/VIla (coagulation factor VIla) signaling pathway promoted angiogenesis ${ }^{29}$. CD133 is one of the key biomarkers for isolation and characterization of stem cells, and it can be found in a few cells including epithelial cell membrane ${ }^{30}$. $\mathrm{CD} 133^{+}$cells are known to have regenerative properties including promoting cell differentiation and enhancing angiogenesis ${ }^{31,32}$ and have been used in a few clinical trials such as ischemia and hepatic fibrosis $^{33,34}$. Similarly found, MSC-derived EV containing CD133 epitope promoted recovery in a moue model of ischemia reperfusion renal injury, proving that $E V$ therapeutics might be superior to cell-based therapy in terms of safety and versatility 35 .

We demonstrated that the term hAEC-EVs, but not preterm hAEC-EVs, were efficacious in mitigating lung injury in a mouse model of experimental BPD. Specifically, we observed an increased tissue-to-air space ratio, secondary septal crest density, and small pulmonary vessel numbers suggesting that term hAECEVs may protect against the classical alveolar simplification associated with postnatal hyperoxia. This improvement in lung structure was accompanied by a reduction in lung inflammation with specific changes in IL-1 $\beta$, TNF-a, LIF, MCP-1, MIP-2 and GM-CSF levels. Similar to term hAEC treatment, term hAEC-EV administration prevented peripheral pulmonary artery muscularization, and further prevented pulmonary hypertension and right ventricle hypertrophy, and improved lung function as long-term outcome. Additionally, term hAEC-EV treatment was associated with increased numbers of AT2 cells, but without affecting the BASC population. This suggests that term hAEC-EVs may improve lung tissue regeneration either through stimulating the local lung stem cell AT2 niche or promoting the maturation of BASCs to AT2 cells.

On PND 7 and 14, the tissue-to-air space ratio and secondary septal crest percentage were significantly decreased in the injured group compared to the control group, and we observed that both term hAEC and term hAEC-EV treatment brought the percentages back to healthy levels. As angiogenesis promotes 
normal lung alveolar development and contributes to the maintenance of alveolar structure ${ }^{36}$, BPD animals with simplified lung structure had fewer pulmonary vessels. Our previous studies showed that only term but not preterm hAECs derived conditioned media supported vascular tubule formation ${ }^{25}$. This supports the findings in our current study where only term hAECs and term hAEC-EVs protected against the loss of small pulmonary vessels.

When observing another characteristic in BPD, lung inflammation, we found that term hAEC-EVs exerted anti-inflammatory effects by PND7. The elevated levels of IL-1 $\beta$ and TNF- $\alpha$ caused by the injury were diminished by either term hAECs, term hAEC-EVs or preterm hAEC-EVs. IL-1 $\beta$ and TNF- $\alpha$ are mainly secreted from activated macrophages and monocytes ${ }^{37}$, which we had previously shown to be reduced by $\mathrm{hAEC}{ }^{23}$. It is thus possible that term EVs may also modulate macrophage function. Along similar lines, MSC-derived EVs reportedly polarized macrophages from M1 to M2 phenotype in vitro ${ }^{38}$, suppressed macrophage pulmonary infiltration in response to hypoxic lung injury ${ }^{39}$, and attenuated burn-induced inflammation through reducing the expression of IL-1 $\beta$ and TNF-a in mice ${ }^{40}$. In addition, we also found in vitro that preterm hAEC-EVs suppressed T cell proliferation more significantly than term hAEC-EVs and that term hAEC-EVs improved macrophage phagocytosis more significantly than preterm hAEC-EVs. Similarly, MSC-derived EVs have also been shown to restore T cell function disrupted by neonatal hypoxia ${ }^{41}$. This data suggests that impact may be due to direct effect of EV cargo on macrophages.

The cytokine levels of GM-CSF, MCP-1, MIP-2 and LIF were significantly higher in injured animals by PND14, as previously reported ${ }^{23}$. Macrophages and monocytes are the major cell types secreting MIP-2 (IL-8 in human) and MCP-1 during immune response. MCP-1 regulates monocyte migration and infiltration, and recruit and direct macrophage movement ${ }^{42}$, and MIP-2 is a neutrophil chemoattractant ${ }^{43}$. It has been reported that alveolar macrophages secrete MIP-2 and MCP-1 during lung inflammation 44,45 , and elevated levels of MCP-1 and IL-8 were reported in lavage fluid of BPD-affected infants ${ }^{46}$. Additionally, GM-CSF is a known macrophage activator ${ }^{47}$, and LIF is known to potentiate macrophage aggregation and activation ${ }^{48}$. In this study we found that similar to term hAEC treatment, term hAEC-EVs reduced the above cytokines to control levels. It is likely that term hAEC-EVs reduced lung inflammation through modulating macrophage and neutrophil populations as $\mathrm{we}^{49}$ and others ${ }^{50,51}$ have shown previously. However, preterm hAEC- EVs only reduced GM-CSF level to normal, leaving IL-1 $\beta$, MCP-1, MIP-2 and LIF at higher levels than control and treated animals.

In this study, we found that the percentage of BASC or AT2 cells were unchanged in the injured group. This correlates with previous studies that have shown that hyperoxia at 75\% oxygen had no effect on BASC proliferation ${ }^{52}$. Surprisingly, unlike hAECs, term hAEC-EVs had no effect on BASC numbers, but significantly increased the percentage of AT2 on PND7 and PND14. The increase in AT2 correlated with the improvement in lung structure, which may have resulted in activation, proliferation and differentiation into AT1 to improve tissue-to-air space ratio. Some researchers have suggested that there are different mechanisms involved in AT2 and BASC proliferation and differentiation ${ }^{53-56}$. The observation that term 
hAEC-EVs increased AT2 numbers while term hAECs increased BASC numbers could therefore be attributed to differential activation of signaling pathways. However, neither BASC nor AT2 proliferation were observed in preterm hAEC-EV treated group.

In our experimentally induced BPD animals, we observed thickened and muscularized pulmonary vessels on PND14, pulmonary hypertension, right ventricle hypertrophy, and declined lung function with increased resistance and increased compliance. Similar to the term hAEC administration, term hAEC-EVs prevented early pulmonary vascular muscularization and later on secondary pulmonary hypertension. Kourembanas and her colleagues induced BPD injury in mice by exposing neonates to $75 \%$ oxygen for a week, they also observed pulmonary vascular remodeling, increased lung capacity, and elevated right ventricular systolic pressure, and found that both bone marrow derived MSCs (BM-MSC) and Wharton's jelly MSCs (WJ-MSCs) derived EVs ameliorated vascular remodeling and improved lung function ${ }^{57,58}$. It is worth noting that although EVs do not package all the factors that cells contain, this study implies that term hAEC-EVs may carry sufficient bioactive material to exert reparative effects. This is supported by studies that showed MSC-EVs had reparative effects, but EV-depleted MSC conditioned media had no effect in a few preclinical models including hypoxic-induced pulmonary hypertension and cardiotoxin induced muscle injury ${ }^{59,60}$. From what mentioned above, Kourembanas and her colleagues treated BPD mice with EVs derived from different sources of MSCs (BM-MSCs and WJ-MSCs) and found both achieved the same therapeutic outcomes. This suggested that EVs from different cell sources could transport effective cargo to exert efficacy. Future experiments targeting specific cargo like microRNA (miRNA) have shown enormous promise for the development of therapeutic agents for human diseases like BPD ${ }^{61,62}$.

Proteomic analysis revealed that EV cargo from term and preterm hAECs were significantly different. Proteins that were more highly expressed in term hAEC-EVs were enriched in several pathways associated with functional characteristics observed in our current study. The extracellular matrix (ECM) organization pathway is crucial to stem cell lineage specification, and cell migration and proliferation through its dynamic regulation of the microenvironment ${ }^{63}$. Cell junction organization pathway and adherents junction interactions pathway are cell-cell or cell-ECM contacts that are required for cell survival, differentiation, and migration ${ }^{64}$. The ECM homeostasis is important in normal lung development, and collagen is one of the major compositions of the $\mathrm{ECM}^{65,66}$. Increased gene expression level of collagen alpha-1 (cola1) was detected in neonatal mice and aided the process of alveolar development ${ }^{67}$. Integrinmediated cell-ECM interactions are known to play an important role in normal lung development too, and higher expression levels of integrin alpha1, 2, 6 and beta1 were reported in the bronchial and alveolar epithelium during the alveolar stage of lung development ${ }^{68,69}$. Indeed, cola1 and integrin alpha6 were highly expressed in term hAEC-EVs compared to preterm hAEC-EVs. Among the proteins that were significantly higher in term hAEC-EVs, 15 proteins were associated with extracellular matrix organisation, 9 proteins were associated with cell junction organisation, and 5 proteins were associated with integrin cell surface interactions. This indicated that term hAEC-EVs may support normal lung development through these pathways. In the pathways associated with proteins that were highly expressed in the 
preterm hAEC-EVs, the top three pathways are involved in the immune system, innate immune system and developmental biology which are associated with proteins 19, 13 and 12, respectively. This suggests that preterm hAEC-EVs may play a role in inflammation. What we found interesting is that Axon guidance protein was highly expressed in both term and preterm EVs, axon guidance proteins guide growing axons during development and control structural plasticity of synaptic connections. Changes in expression or function of these proteins induces pathological changes in neural circuits that predispose to, or cause, neurological diseases. This is an important finding in that EV therapy would be beneficial for preterm babies born with BPD who also develop cerebral palsy ${ }^{70}$, as axonal injury is one of the hallmarks of cerebral palsy. Taken together, the significant differences in EVs protein cargo and the pathways that they are enriched in likely explain the functional differences between preterm and term hAEC-EVs.

We have previously shown that preterm hAECs had limited reparative effect in bleomycin induced lung injury compared to term hAECs ${ }^{71}$. Hence, we did not assess the efficacy of preterm hAECs in treating BPD and expectedly, we show that preterm hAEC-EVs did not have significant therapeutic effect in this BPD disease setting. We think that prematurity and complications associated with preterm birth may have influenced hAEC function and consequently the EV cargo and their functional potency. When bone marrow derived MSCs were preconditioned with 95\% oxygen, the conditioned media contained higher levels of antioxidant stanniocalcin-1 and was more benefit for hyperoxia induced lung injury rats compared to normal cultured MSC conditioned media ${ }^{72}$, which implies the changes of cargo in cell secretomes impacts their functional potency. However, Bhandari et al reported the MSC EVs that derived from umbilical cords of 25-30 weeks of gestational age, exerted reparative effect in 95\% hyperoxia induced BDP mouse model by improving lung structure and function ${ }^{73}$. Another study showed that the placental EVs derived from maternal peripheral plasma, had declined bioactivity (endothelial cell migration area) in late pregnancy compared to early pregnancy ${ }^{74}$. However, this may not reflect the property of fetal stem/stem-like cells derived EVs, as EVs derived from maternal peripheral plasma is a mixture of EVs from maternal and fetal origin, and factors such as secretion of EVs and placental perfusion need to be considered.

\section{Conclusions}

Taken together, this is the first study to show that EVs isolated from term and preterm placenta have different cargo and functional potency despite sharing similar characterization for size distribution, morphology and exosomal markers. This study also demonstrates that term hAEC-EVs, but not preterm hAEC-EVs, have therapeutic efficacy in a mouse model of BPD-like lung injury. Term hAEC-EVs improved lung structure, reduced lung inflammation, and had long term benefit in improving lung function, and preventing pulmonary hypertension and right ventricle hypertrophy.

There are increasing interests in EV application as a cell-free treatment. Compared to cell therapy, EVbased therapeutics have a number of advantages. As EVs are vesicles, the risks associated with administering live cells such as micro-vessel occlusion ${ }^{75}$, infusion toxicities and ectopic tissue 
formation ${ }^{76}$, are avoided. Furthermore, EVs may be stabilised for long term storage ${ }^{77}$, thereby enabling easier storage and low cost cold storage solutions for international distribution ${ }^{78}$. The interest in the commercial development of therapeutics EVs has grown from about 3 companies (Caprocor Inc., ReNeuron Group PLC and Anosys Inc.) in 2014 to $>30$ companies currently ${ }^{79}$. There are 20 registered clinical trials on NIH website applying EVs as a therapy. It is worth noting that donor acceptance criteria and potency assays are very crucial to developing efficacious EV therapies. The International Society of Extracellular Vesicles (ISEV) released a paper regarding EV therapeutics in clinical trials in 2015, describing major considerations for the production of EV-based therapeutics. These include the characterization of the EV cell source, isolation and storage methods, quality control and in vivo analyses of EV potency ${ }^{80}$. The current study has unveiled the therapeutic differences between term and preterm hAEC-EVs in the setting of experimentally induced BPD, and this could be due to the different cargo they carry which may have different functional potencies even if they bear similar characteristics. Further studies on the progenitor/stem/stem-like cells derived from gestational tissues of pregnancy complications are needed to uncover how pregnancy complications affect their therapeutic potential.

\section{Abbreviations}

ALB: Albumin; a-SMA: alpha-smooth muscle actin; ANOVA: Analysis of variance; APO Apolipoproteins; BCA Bicinchoninic acid; BM Bone marrow; BASCs: Bronchioalveolar stem cell; BPD: Bronchopulmonary dysplasia; CTV: CellTrace Violet; cola1: Collagen alpha-1; E: Embryonic; EPCs: Endothelial progenitor cells; ELISA: Enzyme-linked immunosorbent assay; ECM: Extracellular matrix; EVs: Extracellular vesicles; FACS: Fluorescence-activated single cell sorting; FiO2: Fraction of Inspired Oxygen; GM-CSF: Granulocytemacrophage colony-stimulating factor; H\&E: Haematoxylin and eosin; HSP: Heat shock proteins; HSCs/HPCs: Hematopoietic stem/progenitor cells; hAECs: Human amniotic epithelial cells; IL: Interleukin; ISEV: International Society of Extracellular Vesicles; LIF: Leukemia inhibitory factor; LPS:

Lipopolysaccharide; MIP: Macrophage inflammatory protein; MSCs: Mesenchymal stem/stromal cells; miRNA: microRNA; MISEV: Minimal Information for Studies of Extracellular Vesicles; MCP: Monocyte chemoattractant protein; NTA: Nanoparticle tracking analysis; NIH: National Institute of Health; PMA: Paramethoxyamphetamine; PND: Postnatal day; PV loop: Pressure-volume loop; PAT: Pulmonary artery acceleration time; PET: Pulmonary artery ejection time; RANTES Regulated upon activation, normal T cell expressed and presumably secreted; Crs: Respiratory system compliance; Rrs: Respiratory system resistance; RVAWT: Right ventricle anterior wall thickness; SEM: Standard error of mean; SDCBP: Syntenin binding protein; TF: Tissue factor; TEM: Transmission electron microscopy; TNF-a: Tumour necrosis factor-alpha; AT2s: Type II alveolar cells; UC-MSCs: Umbilical cord derived mesenchymal stromal/stem cells; VEFG: Vascular endothelial growth factor; vWF: von Willebrand Factor; WJ: Wharton's jelly.

\section{Declarations}




\section{Ethics approval and consent to participate}

All patients gave written informed consent in accordance with human research ethics guidelines set out by Monash Health. Access to human tissues was obtained from The Ritchie Centre's Tissue Bank (approved HREC No. 01067B, No. 12223B). All animal experiments were approved by the Monash University Animal Ethics Committee (MMCA 2015/06).

\section{Consent for publication}

Not applicable

\section{Availability of data and material}

All data generated or analysed during this study are included in this published article.

\section{Author Contribution}

$D Z, M K, M B, A M, S C, D G, R S$ and RL performed research, collected and analysed data presented in this manuscript. DZ and RL wrote the manuscript. DZ, EMW and RL conceived and designed the research. TY, GK and RH helped with manuscript preparation.

\section{Acknowledgements}

The authors would like to acknowledge the animal house staff for their assistance with animal work; and the Monash Health Translational Precinct technology platform for data generation.

\section{Disclosure Statement}

The authors have no conflicts to declare.

\section{References}

1. Colao IL, Corteling R, Bracewell D, Wall I. Manufacturing Exosomes: A Promising Therapeutic Platform. Trends Mol Med. 2018 Mar;24(3):242-56.

2. Miki T. Stem cell characteristics and the therapeutic potential of amniotic epithelial cells. Am J Reprod Immunol. 2018 Oct;80(4):e13003. 
3. Möbius MA, Thébaud B. Cell therapy for bronchopulmonary dysplasia: promises and perils. Paediatric respiratory reviews. 2016;20:33-41.

4. Chaubey S, Thueson S, Ponnalagu D, Alam MA, Gheorghe CP, Aghai Z, et al. Early gestational mesenchymal stem cell secretome attenuates experimental bronchopulmonary dysplasia in part via exosome-associated factor TSG-6. Stem cell research \& therapy. 2018;9(1):1-26.

5. Li J, Yawno T, Sutherland A, Loose J, Nitsos I, Allison BJ, et al. Term vs. preterm cord blood cells for the prevention of preterm brain injury. Pediatric Research. 2017;82(6):1030-8.

6. Willis GR, Fernandez-Gonzalez A, Anastas J, Vitali SH, Liu X, Ericsson M, et al. Mesenchymal stromal cell exosomes ameliorate experimental bronchopulmonary dysplasia and restore lung function through macrophage immunomodulation. American journal of respiratory and critical care medicine. 2018;197(1):104-16.

7. Yeo Y, Wee R. Efficiency of exosome production correlates inversely with the developmental maturity of MSC donor. 2013.

8. Hodges RJ, Jenkin G, Hooper SB, Allison B, Lim R, Dickinson H, et al. Human amnion epithelial cells reduce ventilation-induced preterm lung injury in fetal sheep. American journal of obstetrics and gynecology. 2012 May;206(5):448 e8-15.

9. Hodges RJ, Jenkin G, Hooper SB, Allison B, Lim R, Dickinson H, et al. Human amnion epithelial cells reduce ventilation-induced preterm lung injury in fetal sheep. YMOB. 2012 Jun 01;206(5):448.e8-e15.

10. Vosdoganes P, Lim R, Koulaeva E, Chan ST, Acharya R, Moss TJ, et al. Human amnion epithelial cells modulate hyperoxia-induced neonatal lung injury in mice. Cytotherapy. 2013;15(8):1021-9.

11. Tan JL, Chan ST, Lo CY, Deane JA, McDonald CA, Bernard CC, et al. Amnion cell-mediated immune modulation following bleomycin challenge: controlling the regulatory $T$ cell response. Stem Cell Res Ther. 2015 Jan 29;6:8.

12. Murphy SV, Shiyun SC, Tan JL, Chan S, Jenkin G, Wallace EM, et al. Human Amnion Epithelial Cells Do Not Abrogate Pulmonary Fibrosis in Mice With Impaired Macrophage Function. Cell Transplantation. 2012 Jul 01;21(7):1477-92.

13. Morandi F, Horenstein AL, Quarona V, Faini AC, Castella B, Srinivasan RC, et al. Ectonucleotidase Expression on Human Amnion Epithelial Cells: Adenosinergic Pathways and Dichotomic Effects on Immune Effector Cell Populations. J Immunol. 2019 Feb 1;202(3):724-35.

14. Hodge A, Lourensz D, Vaghjiani V, Nguyen H, Tchongue J, Wang B, et al. Soluble factors derived from human amniotic epithelial cells suppress collagen production in human hepatic stellate cells. Cytotherapy. 2014;16(8):1132-44.

15. Malhotra A, Lim R, Mockler JC, Wallace EM. Two-year outcomes of infants enrolled in the first-inhuman study of amnion cells for bronchopulmonary dysplasia. Stem cells translational medicine. 2020;9(3):289-94.

16. Lim R, Malhotra A, Tan J, Chan ST, Lau S, Zhu D, et al. First-in-human administration of allogeneic amnion cells in premature infants with bronchopulmonary dysplasia: a safety study. Stem cells translational medicine. 2018;7(9):628-35. 
17. Baker EK, Malhotra A, Lim R, Jacobs SE, Hooper SB, Davis PG, et al. Human amnion cells for the prevention of bronchopulmonary dysplasia: a protocol for a phase I dose escalation study. BMJ open. 2019;9(2):e026265.

18. Zhu D, Kusuma GD, Schwab R, Chan ST, Tan J, Saad MI, et al. Prematurity negatively affects regenerative properties of human amniotic epithelial cells in the context of lung repair. Clin Sci (Lond). 2020 Oct 30;134(20):2665-79.

19. Murphy S, Rosli S, Acharya R, Mathias L, Lim R, Wallace E, et al. Amnion epithelial cell isolation and characterization for clinical use. Current protocols in stem cell biology. 2010 Apr;Chapter 1:Unit 1E 6.

20. Tan JL, Lau SN, Leaw B, Nguyen HPT, Salamonsen LA, Saad MI, et al. Amnion Epithelial Cell-Derived Exosomes Restrict Lung Injury and Enhance Endogenous Lung Repair. Stem Cells Transl Med. 2018 Feb;7(2):180-96.

21. Thery C, Witwer KW, Aikawa E, Alcaraz MJ, Anderson JD, Andriantsitohaina R, et al. Minimal information for studies of extracellular vesicles 2018 (MISEV2018): a position statement of the International Society for Extracellular Vesicles and update of the MISEV2014 guidelines. J Extracell Vesicles. 2018;7(1):1535750.

22. Paule S, Nebl T, Webb Al, Vollenhoven B, Rombauts LJ, Nie G. Proprotein convertase $5 / 6$ cleaves platelet-derived growth factor $A$ in the human endometrium in preparation for embryo implantation. Molecular human reproduction. 2015;21(3):262-70.

23. Zhu D, Tan J, Maleken AS, Muljadi R, Chan ST, Lau SN, et al. Human amnion cells reverse acute and chronic pulmonary damage in experimental neonatal lung injury. Stem cell research \& therapy. 2017;8(1):257.

24. Vosdoganes P, Hodges RJ, Lim R, Westover AJ, Acharya RY, Wallace EM, et al. Human amnion epithelial cells as a treatment for inflammation-induced fetal lung injury in sheep. American journal of obstetrics and gynecology. 2011 Aug;205(2):156 e26-33.

25. Zhu D, Muljadi R, Chan ST, Vosdoganes P, Lo C, Mockler JC, et al. Evaluating the Impact of Human Amnion Epithelial Cells on Angiogenesis. Stem Cells International. 2016;2016.

26. Bian S, Zhang L, Duan L, Wang X, Min Y, Yu H. Extracellular vesicles derived from human bone marrow mesenchymal stem cells promote angiogenesis in a rat myocardial infarction model. Journal of molecular medicine. 2014;92(4):387-97.

27. Cosenza S, Ruiz M, Toupet K, Jorgensen C, Noël D. Mesenchymal stem cells derived exosomes and microparticles protect cartilage and bone from degradation in osteoarthritis. Scientific reports. 2017;7(1):1-12.

28. Abid Hussein MN, Boing AN, Biro E, Hoek FJ, Vogel GM, Meuleman DG, et al. Phospholipid composition of in vitro endothelial microparticles and their in vivo thrombogenic properties. Thromb Res. 2008;121(6):865-71.

29. Bluff JE, Brown NJ, Reed MW, Staton CA. Tissue factor, angiogenesis and tumour progression. Breast Cancer Res. 2008;10(2):204. 
30. Corbeil D, Marzesco AM, Wilsch-Brauninger M, Huttner WB. The intriguing links between prominin-1 (CD133), cholesterol-based membrane microdomains, remodeling of apical plasma membrane protrusions, extracellular membrane particles, and (neuro)epithelial cell differentiation. FEBS Lett. 2010 May 3;584(9):1659-64.

31. Kamei N, Kwon SM, Alev C, Nakanishi K, Yamada K, Masuda H, et al. Ex-vivo expanded human bloodderived CD133+ cells promote repair of injured spinal cord. J Neurol Sci. 2013 May 15;328(1-2):4150 .

32. Kijima Y, Ishikawa M, Sunagawa T, Nakanishi K, Kamei N, Yamada K, et al. Regeneration of peripheral nerve after transplantation of CD133+ cells derived from human peripheral blood. J Neurosurg. 2009 Apr;110(4):758-67.

33. Adler DS, Lazarus H, Nair R, Goldberg JL, Greco NJ, Lassar T, et al. Safety and efficacy of bone marrow-derived autologous CD133+ stem cell therapy. Frontiers in Bioscience (Elite Edition). 2011;3:506.

34. Nikeghbalian S, Pournasr B, Aghdami N, Rasekhi A, Geramizadeh B, Hosseini Asl SM, et al. Autologous transplantation of bone marrow-derived mononuclear and CD133(+) cells in patients with decompensated cirrhosis. Arch Iran Med. 2011 Jan;14(1):12-7.

35. Ranghino A, Bruno S, Bussolati B, Moggio A, Dimuccio V, Tapparo M, et al. The effects of glomerular and tubular renal progenitors and derived extracellular vesicles on recovery from acute kidney injury. Stem cell research \& therapy. 2017;8(1):1-15.

36. Th eacute baud B. Angiogenesis in Lung Development, Injury and Repair: Implications for Chronic Lung Disease of Prematurity. Neonatology. 2007;91(4):291-7.

37. Jang C-H, Choi J-H, Byun M-S, Jue D-M. Chloroquine inhibits production of TNF- $a$, IL-1 $\beta$ and IL-6 from lipopolysaccharide-stimulated human monocytes/macrophages by different modes. Rheumatology. 2006;45(6):703-10.

38. Zhang B, Yin Y, Lai RC, Tan SS, Choo ABH, Lim SK. Mesenchymal stem cells secrete immunologically active exosomes. Stem cells and development. 2013;23(11):1233-44.

39. Lee C, Mitsialis SA, Aslam M, Vitali SH, Vergadi E, Konstantinou G, et al. Exosomes mediate the cytoprotective action of mesenchymal stromal cells on hypoxia-induced pulmonary hypertension. Circulation. 2012;126(22):2601-11.

40. Li X, Liu L, Yang J, Yu Y, Chai J, Wang L, et al. Exosome Derived From Human Umbilical Cord Mesenchymal Stem Cell Mediates MiR-181c Attenuating Burn-induced Excessive Inflammation. EBioMedicine. 2016.

41. Reis M, Willis GR, Fernandez-Gonzalez A, Yeung V, Taglauer E, Magaletta M, et al. Mesenchymal Stromal Cell-Derived Extracellular Vesicles Restore Thymic Architecture and T Cell Function Disrupted by Neonatal Hyperoxia. Frontiers in immunology. 2021;12:1203.

42. Deshmane SL, Kremlev S, Amini S, Sawaya BE. Monocyte chemoattractant protein-1 (MCP-1): an overview. J Interferon Cytokine Res. 2009 Jun;29(6):313-26. 
43. Al-Alwan LA, Chang Y, Mogas A, Halayko AJ, Baglole CJ, Martin JG, et al. Differential roles of CXCL2 and CXCL3 and their receptors in regulating normal and asthmatic airway smooth muscle cell migration. J Immunol. 2013 Sep 1;191(5):2731-41.

44. Driscoll KE. TNFalpha and MIP-2: role in particle-induced inflammation and regulation by oxidative stress. Toxicol Lett. 2000 Mar 15;112-113:177-83.

45. Chao J, Donham P, van Rooijen N, Wood JG, Gonzalez NC. Monocyte chemoattractant protein-1 released from alveolar macrophages mediates the systemic inflammation of acute alveolar hypoxia. Am J Respir Cell Mol Biol. 2011 Jul;45(1):53-61.

46. Baier RJ, Loggins J, Kruger TE. Monocyte chemoattractant protein-1 and interleukin-8 are increased in bronchopulmonary dysplasia: relation to isolation of Ureaplasma urealyticum. J Investig Med. $2001 \mathrm{Jul} ; 49(4): 362-9$.

47. Shi Y, Liu CH, Roberts Al, Das J, Xu G, Ren G, et al. Granulocyte-macrophage colony-stimulating factor (GM-CSF) and T-cell responses: what we do and don't know. Cell research. 2006;16(2):126-33.

48. Conti P, Barbacane RC, Reale M, Panara MR, Placido FC, Mier JW, et al. Leukocyte inhibitory factor (LIF) potentiates human macrophage aggregation and activation responses to calcium ionophore A23187 and directly induces leukotriene B4 and thromboxane A2 release. Biotechnol Ther. 1993;4(34):239-52.

49. Tan JL, Lau SN, Leaw B, Nguyen HP, Salamonsen LA, Saad MI, et al. Amnion epithelial cell-derived exosomes restrict lung injury and enhance endogenous lung repair. Stem Cells Translational Medicine. 2018;7(2):180-96.

50. Harrell CR, Jovicic N, Djonov V, Arsenijevic N, Volarevic V. Mesenchymal stem cell-derived exosomes and other extracellular vesicles as new remedies in the therapy of inflammatory diseases. Cells. 2019;8(12):1605.

51. Harrell CR, Jovicic N, Djonov V, Volarevic V. Therapeutic use of mesenchymal stem cell-derived exosomes: from basic science to clinics. Pharmaceutics. 2020;12(5):474.

52. Tropea KA, Leder E, Aslam M, Lau AN, Raiser DM, Lee J-H, et al. Bronchioalveolar stem cells increase after mesenchymal stromal cell treatment in a mouse model of bronchopulmonary dysplasia. American Journal of Physiology-Lung Cellular and Molecular Physiology. 2012;302(9):L829-L37.

53. Lee J-H, Bhang DH, Beede A, Huang TL, Stripp BR, Bloch KD, et al. Lung stem cell differentiation in mice directed by endothelial cells via a BMP4-NFATc1-thrombospondin-1 axis. Cell. 2014;156(3):44055 .

54. Moodley Y, llancheran S, Samuel C, Vaghjiani V, Atienza D, Williams ED, et al. Human amnion epithelial cell transplantation abrogates lung fibrosis and augments repair. American journal of respiratory and critical care medicine. 2010;182(5):643-51.

55. Wang Y, Huang C, Chintagari NR, Bhaskaran M, Weng T, Guo Y, et al. miR-375 regulates rat alveolar epithelial cell trans-differentiation by inhibiting Wnt/ $\beta$-catenin pathway. Nucleic acids research. 2013;41(6):3833-44. 
56. Zhang Y, Goss AM, Cohen ED, Kadzik R, Lepore JJ, Muthukumaraswamy K, et al. A Gata6-Wnt pathway required for epithelial stem cell development and airway regeneration. Nature genetics. 2008;40(7):862-70.

57. Willis GR, Fernandez-Gonzalez A, Reis M, Yeung V, Liu X, Ericsson M, et al. Mesenchymal stromal cellderived small extracellular vesicles restore lung architecture and improve exercise capacity in a model of neonatal hyperoxia-induced lung injury. J Extracell Vesicles. 2020 Jul 13;9(1):1790874.

58. Willis GR, Fernandez-Gonzalez A, Anastas J, Vitali SH, Liu X, Ericsson M, et al. Mesenchymal Stromal Cell Exosomes Ameliorate Experimental Bronchopulmonary Dysplasia and Restore Lung Function through Macrophage Immunomodulation. Am J Respir Crit Care Med. 2018 Jan 1;197(1):104-16.

59. Lee C, Mitsialis SA, Aslam M, Vitali SH, Vergadi E, Konstantinou G, et al. Exosomes mediate the cytoprotective action of mesenchymal stromal cells on hypoxia-induced pulmonary hypertension. Circulation. 2012 Nov 27;126(22):2601-11.

60. Nakamura Y, Miyaki S, Ishitobi H, Matsuyama S, Nakasa T, Kamei N, et al. Mesenchymal-stem-cellderived exosomes accelerate skeletal muscle regeneration. FEBS Lett. 2015 May 8;589(11):1257-65.

61. Zhang D, Lee H, Wang X, Rai A, Groot M, Jin Y. Exosome-mediated small RNA delivery: a novel therapeutic approach for inflammatory lung responses. Molecular Therapy. 2018;26(9):2119-30.

62. Zhong X-q, Yan Q, Chen Z-g, Jia C-h, Li X-h, Liang Z-y, et al. Umbilical Cord Blood-Derived Exosomes From Very Preterm Infants With Bronchopulmonary Dysplasia Impaired Endothelial Angiogenesis: Roles of Exosomal MicroRNAs. Frontiers in cell and developmental biology. 2021;9:529.

63. Walker C, Mojares E, Del Rio Hernandez A. Role of Extracellular Matrix in Development and Cancer Progression. Int J Mol Sci. 2018 Oct 4;19(10).

64. Costa P, Parsons M. New insights into the dynamics of cell adhesions. Int Rev Cell Mol Biol. 2010;283:57-91.

65. Ozbek S, Balasubramanian PG, Chiquet-Ehrismann R, Tucker RP, Adams JC. The evolution of extracellular matrix. Mol Biol Cell. 2010 Dec;21(24):4300-5.

66. Lang MR, Fiaux GW, Gillooly M, Stewart JA, Hulmes DJ, Lamb D. Collagen content of alveolar wall tissue in emphysematous and non-emphysematous lungs. Thorax. 1994 Apr;49(4):319-26.

67. Mariani TJ, Reed JJ, Shapiro SD. Expression profiling of the developing mouse lung: insights into the establishment of the extracellular matrix. Am J Respir Cell Mol Biol. 2002 May;26(5):541-8.

68. Coraux C, Delplanque A, Hinnrasky J, Peault B, Puchelle E, Gaillard D. Distribution of integrins during human fetal lung development. J Histochem Cytochem. 1998 Jul;46(7):803-10.

69. Coraux C, Meneguzzi G, Rousselle P, Puchelle E, Gaillard D. Distribution of laminin 5, integrin receptors, and branching morphogenesis during human fetal lung development. Dev Dyn. 2002 Oct;225(2):176-85.

70. Van Marter LJ, Kuban KC, Allred E, Bose C, Dammann O, O'Shea M, et al. Does bronchopulmonary dysplasia contribute to the occurrence of cerebral palsy among infants born before 28 weeks of gestation? Archives of Disease in Childhood-Fetal and Neonatal Edition. 2011;96(1):F20-F9. 
71. Lim R, Chan ST, Tan JL, Mockler JC, Murphy SV, Wallace EM. Preterm human amnion epithelial cells have limited reparative potential. Placenta. 2013 Jun;34(6):486-92.

72. Waszak P, Alphonse R, Vadivel A, lonescu L, Eaton F, Thebaud B. Preconditioning enhances the paracrine effect of mesenchymal stem cells in preventing oxygen-induced neonatal lung injury in rats. Stem Cells Dev. 2012 Oct 10;21(15):2789-97.

73. Chaubey S, Thueson S, Ponnalagu D, Alam MA, Gheorghe CP, Aghai Z, et al. Early gestational mesenchymal stem cell secretome attenuates experimental bronchopulmonary dysplasia in part via exosome-associated factor TSG-6. Stem Cell Res Ther. 2018 Jun 26;9(1):173.

74. Salomon C, Torres MJ, Kobayashi M, Scholz-Romero K, Sobrevia L, Dobierzewska A, et al. A gestational profile of placental exosomes in maternal plasma and their effects on endothelial cell migration. PLoS One. 2014;9(6):e98667.

75. Furlani D, Ugurlucan M, Ong L, Bieback K, Pittermann E, Westien I, et al. Is the intravascular administration of mesenchymal stem cells safe?: Mesenchymal stem cells and intravital microscopy. Microvascular research. 2009;77(3):370-6.

76. Merino-González C, Zuñiga FA, Escudero C, Ormazabal V, Reyes C, Nova-Lamperti E, et al. Mesenchymal Stem Cell-Derived Extracellular Vesicles Promote Angiogenesis: Potencial Clinical Application. Frontiers in physiology. 2016;7.

77. Lorincz AM, Timar Cl, Marosvari KA, Veres DS, Otrokocsi L, Kittel A, et al. Effect of storage on physical and functional properties of extracellular vesicles derived from neutrophilic granulocytes. $J$ Extracell Vesicles. 2014;3:25465.

78. Zhou H, Yuen PS, Pisitkun T, Gonzales PA, Yasuda H, Dear JW, et al. Collection, storage, preservation, and normalization of human urinary exosomes for biomarker discovery. Kidney international. 2006;69(8):1471-6.

79. Lötvall J, Hill AF, Hochberg F, Buzás El, Di Vizio D, Gardiner C, et al. Minimal experimental requirements for definition of extracellular vesicles and their functions: a position statement from the International Society for Extracellular Vesicles. Journal of extracellular vesicles. 2014;3.

80. Lener T, Gimona M, Aigner L, Borger V, Buzas E, Camussi G, et al. Applying extracellular vesicles based therapeutics in clinical trials - an ISEV position paper. J Extracell Vesicles. 2015;4:30087.

\section{Figures}

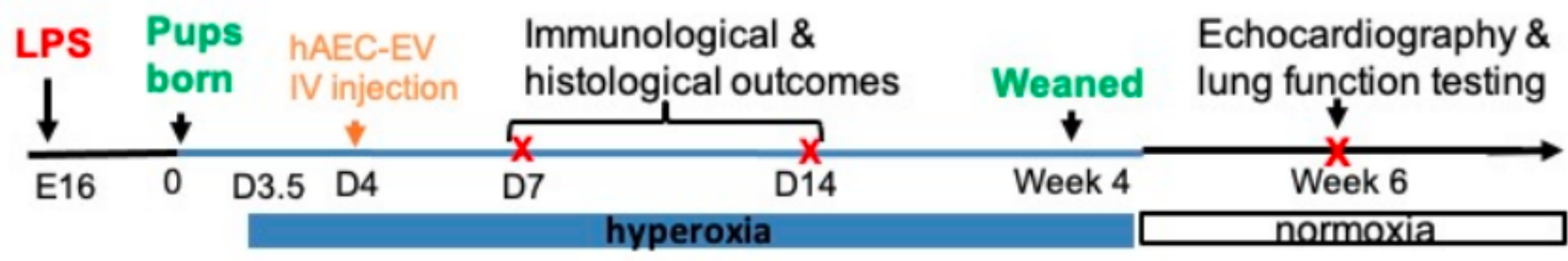




\section{Figure 1}

The flowchart of experimental induced BPD bronchopulmonary dysplasia (BPD) model. C57/BL6 mice were time-mated and on day 16 of pregnancy (E16) injected with either $0.1 \mathrm{mg}$ lipopolysaccharide (LPS) in $5 \mathrm{~mL}$ saline or an equal volume of vehicle into each amniotic sac. Pups were then allowed to deliver naturally at term. The pups were placed either in normoxia or hyperoxia chambers on PND3.5 (12 hours before hAEC treatment). On PND4, either hAECs (human amnion epithelial cells, 100,000 cells) or EVs (term/preterm extracellular vesicles, $10 \mathrm{mg}$ ) or saline was injected intravenously through the superficial temporal vein using the automated microinjection system as per the intra-amniotic injections. One cohort of mouse pups were culled on either PND7 or PND14. Another cohort of mouse pups were kept in hyperoxia chamber until PND28 when they were weaned and transferred to standard housing facilities. Animals were culled at 6 weeks of age. 


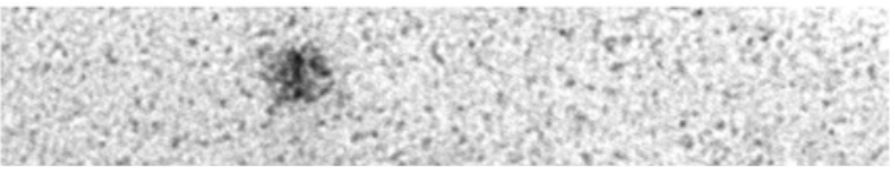

\section{Figure 2}

Characterization of human amnion epithelial cell derived extracellular vesicle (hAEC-EV) ( $n=5)$. (A) The representative transmission electron microscopy image of isolated hAEC-EVs. (B) The representative figure of particle distribution in hAEC-EVs. There was no significant difference in cell viability between term and preterm hAECs (C), and there were no differences in protein yield (D) particle numbers (E), and particle mean size $(\mathrm{F})$ between term and preterm hAEC-EVs. 


\section{Figure 3}

EV surface epitopes detected by MACSPlex $(n=5)$. All hAEC-EVs presented EV common tetraspanins CD 9 (A) and CD63 (B), surface epitope CD105 (C), and epithelial marker CD326 (D). There were no differences in expression levels of these surface epitopes between term EVs and preterm EVs. Term EVs expressed higher levels of CD142 (E) and CD133 (F) compared to preterm EVs. $\left({ }^{*} \mathrm{p}<0.05\right)$
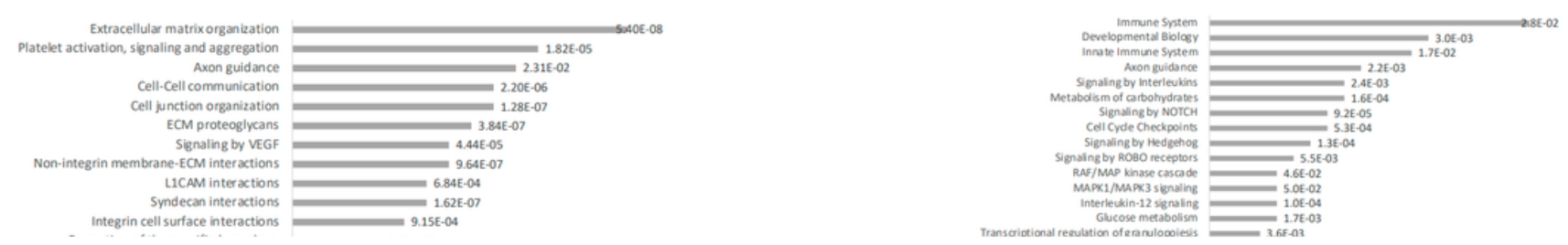

\section{Figure 4}

The pathway analysis of proteins. $(n=5)$. (A) The pathway analysis of 47 proteins that were highly expressed in term EVs compared to preterm EVs with fold change $>20$. There were 19 pathways including extracellular organization (15 proteins related), cell junction organization (9 proteins related), integrin cell surface interaction (4 proteins related), and adherens junction interactions (4 proteins related). (B) The pathway analysis of 35 proteins that were highly expressed in preterm EVs compared to term EVs with fold change $>20$. There were 27 pathways including immune system (13 proteins related), development biology (13 proteins related), innate immune system (12 proteins related), and signaling by Hedgehog ( 6 proteins related). 


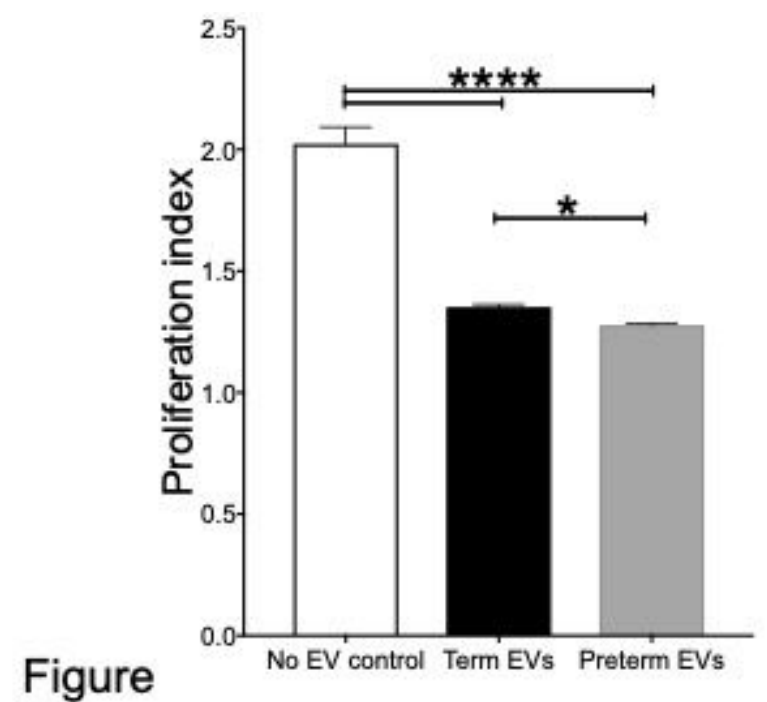

$5 \mathrm{~A}$

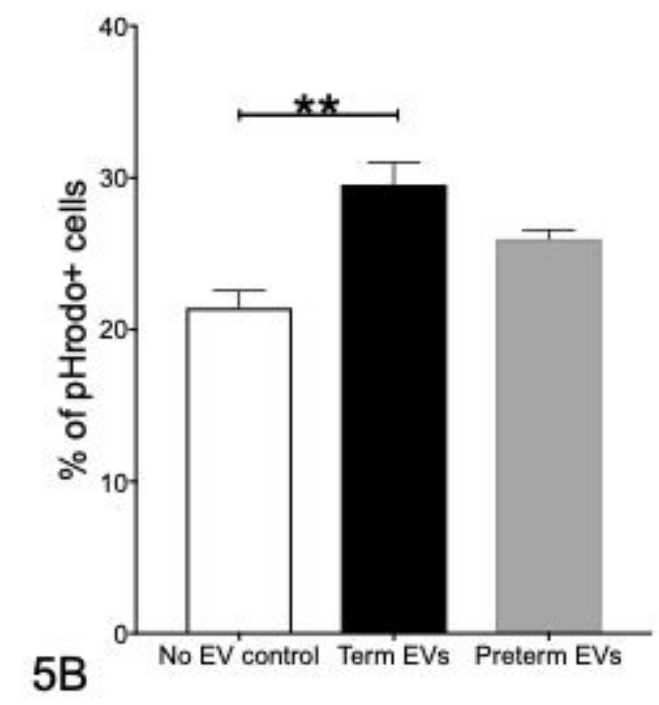

Figure 5

In vitro potency assays $(\mathrm{n}=5)$. (A) Preterm EVs suppressed T cell proliferation more significantly than term EVs with lower proliferation index. (B) Term EVs improved macrophage phagocytosis more significantly than preterm EVs. $\left(* \star \star \star x p<0.0001,{ }^{*} p<0.05\right)$

\section{Figure 6}

Tissue-to-air space ratio on PND 7 and $14(n=6)$. (A) Representative images for H\&E staining (PND7). (B) On PND7, tissue-to-air space ratio was decreased in saline treated injured group compared to control groups. In contrast to hAEC treatment, only term but not preterm EVs improved lung structure. (C) On PND14, tissue-to-air space ratio remained lower in saline treated injured group. Term EV treatment mitigated tissue-to-air space ratio and made it comparable to hAEC treatment group and healthy control group. However, preterm EVs did not improve lung structure. $\left({ }^{*} p<0.05,{ }^{* \star} p<0.01,{ }^{* \star \star} p<0.001\right)$

\section{Figure 7}

Hart's staining on PND7 and $14(n=6)$. (A) Representative images for Hart's staining on PND14. (B) Term hAEC injection improved secondary septal crest density to control levels. (C) Secondary septal crest density was decreased in the saline treated injured group, hAEC and term EV injection normalized it to 
control levels. However, preterm EV treatment did not improve secondary septal crest density. $\left({ }^{\star} p<0.05\right.$, $\left.{ }^{\star *} \mathrm{p}<0.01, * \star \star \mathrm{p}<0.001\right)$

\section{Figure 8}

Changes of inflammatory cytokine levels in mouse lung lysate $(n=6)$. On PND7, IL-1 $\beta(A)$ and TNF- $a(B)$ levels were increased in injured group, and EVs normalized them to control levels, which is comparable to hAEC treatment. On PND14, IL-1 $\beta$ (C) and MIP-2 (D) levels were higher in preterm EV treated group. (E) Levels of RANTES only increased in the hAEC treatment group, but not in the EV group. LIF (F), MCP-1 (G) and GM-CSF $(\mathrm{H})$ levels were increased in the injured group, and both hAECs and term EVs reduced them to control levels. However, levels of LIF and MCP-1 remained higher in the preterm EV treatment group. $\left({ }^{\star} \mathrm{p}<0.05,{ }^{\star *} \mathrm{p}<0.01,{ }^{* * *} \mathrm{p}<0.001\right)$

\section{Figure 9}

Bronchioalveolar stem cells (BASCS) and type II alveolar cells (AT2s) in mouse lungs at PND7 and 14 $(n=6)$. (A) Representative image of pro-SPC (red) and CC10 (green) immunohistochemical staining on mouse lung tissues where arrows point to BASCs (scale bars $=50 \mathrm{~mm})$. (B-C) The average number of BASCs per terminal bronchiole on PND7 (B) and PND14 (C). In contrast to hAEC treatment group, where the average number of BASCs was significantly higher than control group on PND14, it did not change in EV groups on both time points. (D-E) The percentage of AT2 cells was significantly increased in term EV treatment group on both PND7 (D) and 14 (E) compared to control group, which is on contrast to the unchanged AT2 cell percentage in both hAEC and preterm EV treatment groups $\left({ }^{*} p<0.05,{ }^{*} p<0.01\right.$, $\star \star \star * p<0.001, \star \star \star \star p<0.0001)$

\section{Figure 10}

von Willebrand Factor (vWF) immunohistochemistry in mouse lung tissue at PND 7 and $14(n=6)$. (A) Representative images for VWF staining on PND14. (B-C) The average number of vessels per field of view on PND7 (B) and PND14 (C). The average number of vessels with diameter <50 $\mu$ m (black bars) was decreased in saline treated injured mice, which was restored after hAEC and term EV treatment on both 
PND7 and 14. There was no difference in the numbers of larger blood vessels ( $>50 \mu \mathrm{m}$, grey bars) between groups. ( $\left.{ }^{*} p<0.05, * \star p<0.01\right)$

\section{Figure 11}

Smooth muscle alpha-actin (a-SMA) immunofluorescence in mouse lung tissue at PND14 ( $n=6)$. (A) Representative images for a-SMA immunofluorescence in mouse lung tissues at PND14. Scale bars $=50 \mu \mathrm{m}$. Vessels are indicated with white arrows. (B) By PND 14, arterial medial thickness was increased in injured mice. This was mitigated by both hAEC and term EV treatment, but not preterm EV treatment. $\left({ }^{\star} p<0.05,{ }^{* *} p<0.01, * * \star p<0.001,{ }^{* \star * \star} p<0.0001\right)$

\section{Figure 12}

Hematoxylin and eosin (H\&E) staining of mouse lung tissue at week $6(n=6)$. (A) The representative images for H\&E staining on mouse lung tissues by week 6 . Scale bars $=200 \mu \mathrm{m}$. (B) hAEC treatment improved the tissue-to-air space ratio compared to saline treated injured mice, but remained lower than controls. Term EV treatment improved the tissue-to-air space ratio, making it between hAEC treatment group and saline treated group. While preterm EV treatment made no significant difference to the saline

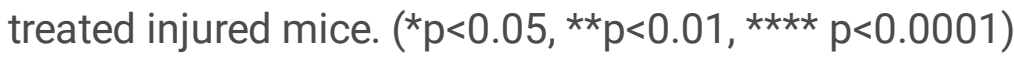

\section{Figure 13}

Invasive lung function test on 6-week old mice $(n=6)$. (A-B) There were no significant differences in the baseline of either respiratory resistance (Rrs, A) or compliance (Crs, B) between groups. (C-D) Compared to control group, Rrs was significantly increased (C) and Crs was significantly decreased (D) at the $100 \mathrm{mg} / \mathrm{ml}$ methacholine dose in the injured group, but this effect was diminished with hAEC treatment and also improved with term EV treatment groups. (E) Pressure volume loop (PV loop) on week 6 . There was a significant upward shift of the PV loop in the injured group compared to the control group. While the PV loop of both hAEC treatment group and term EV treatment group were intermediate to both control and saline treated injured groups, preterm EV treatment had no effect on the position of the PV loop. $\left({ }^{\star} \mathrm{p}<0.05,{ }^{* \star} \mathrm{p}<0.01, * \star * \mathrm{p}<0.001,{ }^{* \star * *} \mathrm{p}<0.0001\right)$ 


\section{Figure 14}

Changes in pulmonary artery flow on 6-week old mice $(n=6)$. (A) Representative images of pulmonary artery flow. (B) Changes of PAT/PET (pulmonary artery acceleration time/ejection time) ratio. Injury decreased the ratio of PAT/PET, which indicated the development of pulmonary hypertension. Both hAEC treatment and term EV treatment restored the ratio back to control level, however, preterm EV treatment group didn't have significant changes. $\left({ }^{*} \mathrm{p}<0.01\right)$

\section{Figure 15}

Changes to right ventricle anterior wall thickness (RVAWT) on 6-week old mice $(n=6)$. (A) Representative images of the RVAWT. (B) The RVAWT increased in the injured mice, while both hAEC treatment and term EV treatment groups, but not preterm EV group, decreased the wall thickness to control levels. $(* \star p<0.01$, $\star \star \star \star \star p<0.0001)$ 